Cold Spring Harbor Symposia on Quantitative Biology

\title{
sci-hub.tw
}

doi:10.1101/sqb.2003.68.179

Cold Spring Harb Symp Quant Biol 2003. 68: 179-188

\section{Positional Identification of Structural and Regulatory Quantitative Trait Nucleotides in Domestic Animal Species}

M. GEORGES and L. ANDERSSON

+ Author Affiliations

This extract was created in the absence of an abstract.

\section{Excerpt}

Although the identification of mutations underlyingmonogenic traits has become nearly trivial, the moleculardissection of multifactorial traits-which include the majority of medically and agronomically important phenotypes—remains a major challenge. Despite the substantial resources that have been allocated to suchefforts-particularly in human-a recent survey reportedonly 30 successful outcomes, all organisms confounded(Glazier et al. 2002). This number has to be comparedwith the more than 1,600 Mendelian traits for which thecausal mutation has been identified in the human only. Asrecently stated by Rutherford and Henikoff (2003), "Thenature of quantitative-trait 